\title{
A study on drug induced Stevens-Johnson Syndrome (SJS), Toxic Epidermal Necrolysis (TEN) and SJS-TEN overlap in a tertiary care hospital of Northeast India
}

\author{
Ratan J. Lihite, ${ }^{1}$ Mangala Lahkar, ${ }^{2}$ Ajoy Borah, ${ }^{3}$ Debeeka Hazarika, ${ }^{4}$ Sukhjinder Singh ${ }^{5}$ \\ 'Department of Pharmacology, ADR Monitoring Centre (Pharmacovigilance Programme of India, IPC-NCC, Ministry of Health \& Family welfare, Gol), Gauhati Medical \\ College \& Hospital, Guwahati-781032, Assam, INDIA. \\ 2Department of Pharmacology \& NIPER-Guwahati, Gauhati Medical College \& Hospital, Guwahati-781032, Assam, INDIA. \\ 3Department of Pharmacology, Gauhati Medical College \& Hospital, Guwahati-781032, Assam, INDIA. \\ ${ }^{4}$ Department of Dermatology \& STD, Gauhati Medical College \& Hospital, Guwahati-781032, Assam, INDIA. \\ ${ }^{5}$ Department of Pharmacy Practice, National Institute of Pharmaceutical Education \& Research (NIPER) (Department of Pharmaceuticals, Ministry of Chemicals and \\ Fertilizers, Gol), Guwahati-781032, Assam, INDIA.
}

\begin{abstract}
Background: Stevens Johnson syndrome (SJS), toxic epidermal necrolysis (TEN) and SJS-TEN overlap are rare, but potentially life-threatening, reactions to medications. From Northeast India, limited epidemiological data is available on these cutaneous drug reactions. Aims: The purpose of this study was to assess the drug-induced SJS/TEN cases of a tertiary care hospital of Northeast India. Methods: This cross sectional study was conducted in Gauhati Medical College and Hospital of Guwahati city, Northeast India. WHO-UMC probability assessment system and ALDEN were used for causality assessment of reported SJS/TEN cases. Results are presented in percentage; mean (standard deviation), and median (interquartile range difference). Results: Forty five cases of drug induced SJS $(42.22 \%)$, TEN $(55.55 \%)$ \& SJS-TEN (2.22\%) overlap were reported. Out of these, 25 patients were males and 20 patients were females. The mean age of the patients was 29.69 (17.57) year. The median time duration between drug intake and onset of symptoms for SJS/TEN was 25(4) and the median duration of hospital stay by patients was 13(8). The most offending groups of drugs were antimicrobials $(35.55 \%)$, followed by anticonvulsants $(28.89 \%)$, antipyretics $(17.78 \%)$, and nonsteroidal anti-inflammatory drugs (6.67\%). In individual drug category, paracetamol $(17.77 \%)$ and phenytoin $(15.55 \%)$ were among the commonly reported offending drugs. Altered liver
\end{abstract}

function test was commonly reported complication in SJS/TEN patients. As per ALDEN, drug causality was very probable in 16 cases whereas by WHO-UMC assessment system, 1 case was certain while 29 cases were probable. Limitations: Statistical significance among the variables was not shown and large study sample may be required for interpretation of results to arrive at a definite conclusion. Conclusions: Antimicrobials, anticonvulsants, and antipyretics were commonly reported group of offending drugs to cause SJS/TEN in patients. Paracetamol \& phenytoin were commonly reported individual offending drugs.

Key words: SJS, TEN, Northeast India, Paracetamol, Anticonvulsants.

Correspondence :

Ratan J. Lihite,

Department of Pharmacology, ADR Monitoring Centre, Gauhati Medical College and Hospital, Guwahati-781032, Assam, INDIA.

Phone on: +91 9706143510

E-mail: r.lihite@yahoo.com

DOI: $10.5530 /$ jyp.2016.2.18

\section{INTRODUCTION}

Adverse drug reactions (ADR) accounts 6\% of the total hospital admission, increases economic burden on healthcare system, results into withdrawal of drugs from market and death. ${ }^{1,2}$ Among various ADR, cutaneous drug reactions mainly Stevens-Johnson syndrome (SJS) and Toxic Epidermal Necrolysis (TEN) are rare but potentially fatal reactions endangers patient's life. These reactions are characterized by mucocutaneous tenderness, typically hemorrhagic erosions, erythema with more or less severe epidermal detachment presenting as blisters and areas of denuded skin. ${ }^{3}$ In addition to the severe skin symptoms, both diseases are often accompanied by complications in numerous organs, such as the liver, kidney, and lung. Patient may initially present with SJS, which subsequently evolves into TEN or SJS-TEN overlap. Diagnosis mainly relies on clinical signs and histopathology of skin lesions. The exact pathogenesis of SJS and TEN remains to be elucidated but apoptotic mechanisms, including involvement of cytotoxic T cells, tumor necrosis factor (TNF)- $\alpha$, and Fas (CD95), Fas ligand (FasL) interaction are considered to be relevant to these diseases. ${ }^{4}$

As reported, the rate of occurrence of SJS \& TEN to medications is low. ${ }^{2,5}$ However, drugs are most commonly implicated for SJS, TEN and SJS-TEN overlap and other possible causes are infections, immunizations, environment chemicals and radiation therapy. ${ }^{2,5}$ The incidence of SJS ranges from 1.2 to 6 patients per million per year, while for TEN it ranges from 0.4 to 1.2 patients per million per year. ${ }^{6}$ The mortality for SJS varies from $3 \%$ to $10 \%$ and for TEN from $20 \%$ to $40 \%{ }^{7}$

It was observed that still there is lack of studies on these cutaneous disorders from Northeast region of India as compare to other part of country. ${ }^{2}$ This cross sectional study was conducted to assess drug-induced SJS, TEN and SJS-TEN overlap cases reported from a tertiary care hospital situated at Guwahati, Northeast India. In our study, cases with widespread purpuric macules and epidermal detachment below $10 \%$ are called SIS. Those with cutaneous detachment between $10 \%$ and $30 \%$ are called transitional SJS-TEN, and those with more than 30\% epidermal detachment are designed TEN. ${ }^{8}$

\section{METHODS}

The permission from institutional ethical committee of Gauhati Medical College \& Hospital, Guwahati (Northeast India) was obtained to conduct this study. Patients admitted between January 2011 and December 2014, and diagnosed with SJS, SJS/TEN overlap or TEN were identified from the hospital. The diagnosis of SJS, TEN, and SIS-TEN overlap was done according to the criteria laid down by Bastuji-Garin et al. ${ }^{8}$ These cases were reported from inpatient departments of dermatology, paediatrics, gynaecology, medicine, psychiatry, neurology, haematology and intensive treatment unit of hospital. Those patients were excluded who complained of only symptoms without visible skin lesions, those who 
could not recall the name of the consumed drug, and other whose lesions turned out to be disease related (e.g. viral exanthems, rash of rickettsial infections, and collagen vascular disease) on closer examination. The patients who reported to have consumed ayurvedic and homeopathic drugs were also excluded as the herbal ingredients could not be identified in their case.

We have collected data of those patients who had following details in a predesigned suspected ADR reporting form of Pharmacovigilance Programme of India. ${ }^{9}$ Collected data included patient's age \& sex, duration of hospital stay, the time period between the drug component intake \& onset of eruption, suspected drug (drug notoriety), dechallenge and prechallenge/rechallenge of suspected drug, indication, and other complications. Drug that has been taken within four weeks preceding the onset of symptoms was considered as culprit drug.

In present study, WHO-UMC probability assessment system and algorithm for assessment of drug causality in Epidermal Necrolysis (ALDEN) ${ }^{10}$ were followed for assessing the causality of reported ADR cases. WHOUMC probability assessment system is generally used for drug causality assessment under Pharmacovigilance Programme of India; proposed by the WHO collaborating centre for international drug monitoring, the Uppsala Monitoring Centre (UMC), Sweden. ${ }^{9}$ While using ALDEN, the index day (time lag from initiation of drug intake to onset of reaction) for all the reported cases were suggestive $(+3)$ except one whose index day was 29 , which was categorized as compatible $(+2)$. The probability that the drug was present in the body at the time of the onset of the reaction was considered definite (0) for all the reported SJS/TEN cases. The suspected drug was withdrawn (dechallenge) after the onset of eruptions in all the SIS/TEN cases and prechallenge/rechallenge was not performed in any reported cases. The drug notoriety was performed as per the Euro SCAR study as high risk drug, lower risk drug and the drug of no evidence of association. ${ }^{11}$

Statistical analysis: Descriptive statistics were used for data analysis. Mean (standard deviation) was calculated for normally distributed continuous variable whereas for non-normally distributed continuous variable, results are presented in median (interquartile range difference).

\section{RESULTS}

During study period, 45 cases of drug induced SJS (19/45, 42.22\%), TEN $(25 / 45,55.55 \%) \&$ SJS-TEN $(1 / 45,2.22 \%)$ overlap were reported. Total 25 males and 20 females were reported. Of these, 11 males \& 8 females had SJS while 12 males \& 13 females had TEN. Only 1 male patient of SJS-TEN overlap was reported. The mean age of the patients was 29.69 (17.57) year. The youngest patient was of 4 year old child while oldest was of 83 year old. The median time duration between drug intake and onset of symptoms for SJS/TEN was 25(4) and the median duration of hospital stay by patients was 13(8).

The causality assessment was performed using probability assessment systems of WHO-UMC and ALDEN. Drug causality was very probable in 16 cases by ALDEN whereas as per WHO-UMC assessment system, 1 case was certain while 29 cases were probable (Figure 1 ).

The major group of drugs causing SJS/TEN were antimicrobials $(16 / 45$, $35.55 \%)$, anticonvulsants $(13 / 45,28.89 \%)$, antipyretics $(8 / 45,17.78 \%)$, nonsteroidal anti-inflammatory drugs (NSAIDs) (3/45, 6.67\%), and anticancer drugs $(2 / 45,4.4 \%)$ (Table 1$)$. Besides these, there were cases due to methotrexate, lenalidomide, allopurinol and reboxetine (Table 1).

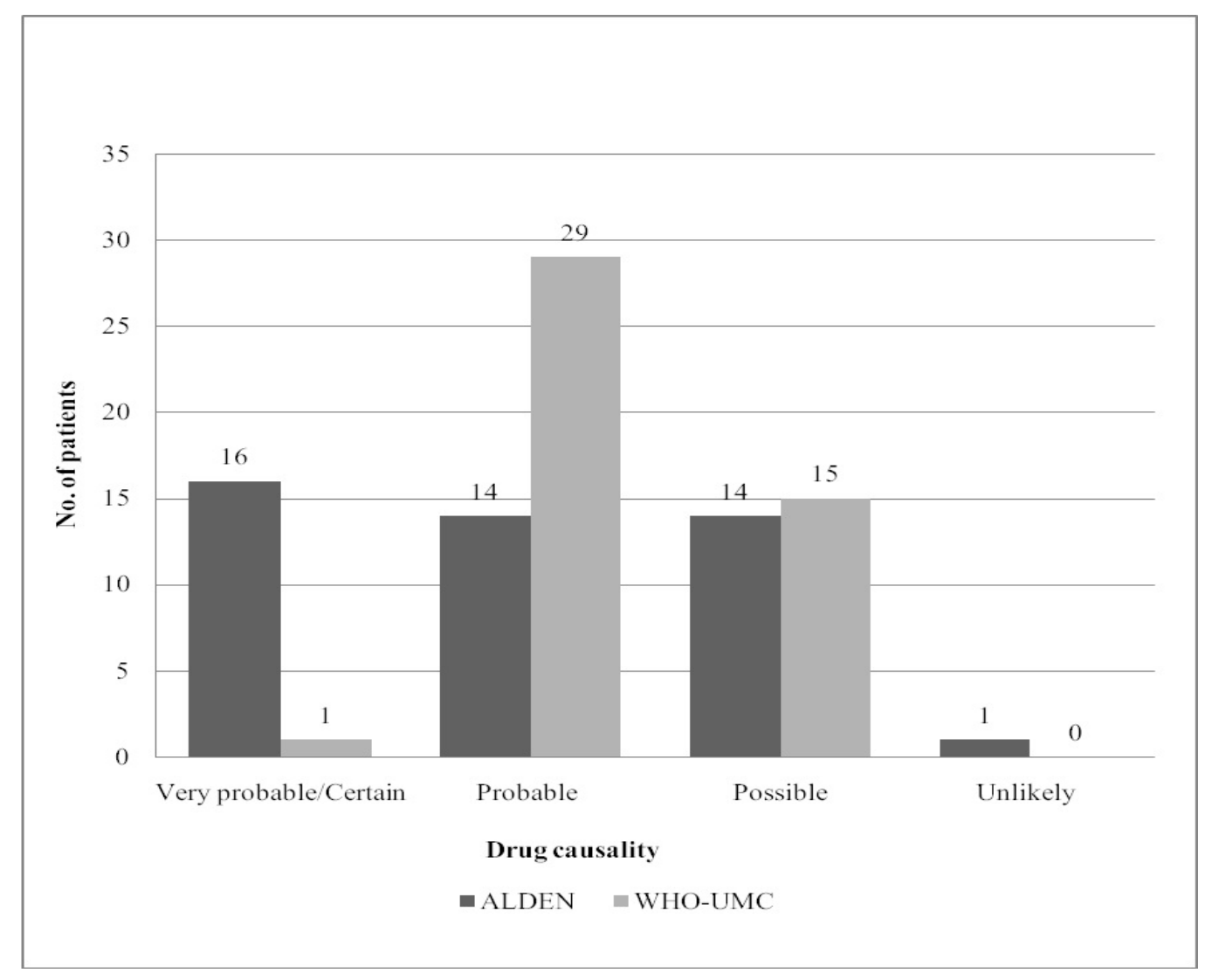

Figure 1: Drug causality assessment using ALDEN and WHO-UMC 
Table 1: Offending drugs with respect to reactions

\begin{tabular}{|c|c|c|c|c|}
\hline Offending drugs & $\begin{array}{l}\text { No. of patients } \\
\text { with SJS }\end{array}$ & $\begin{array}{l}\text { No. of patients } \\
\text { with TEN }\end{array}$ & $\begin{array}{l}\text { No. of patients with } \\
\text { SJS-TEN overlap }\end{array}$ & $\begin{array}{r}\text { Total no. of } \\
\text { patients (\%) }\end{array}$ \\
\hline Antimicrobial drugs & {$[5]$} & [11] & - & $16(35.5 \%)$ \\
\hline levofloxacin (fluoroquinolones) & 1 & - & - & 1 \\
\hline ceftazidime ( $3^{\text {rd }}$ cephalosporins) & 1 & - & - & 1 \\
\hline sulfamethizole (sulfonamides) & - & 1 & - & 1 \\
\hline cefixime ( $3^{\text {rd }}$ cephalosporins) & - & 3 & - & 3 \\
\hline cefuroxime ( $2^{\text {nd }}$ cephalosporins) & - & 1 & - & 1 \\
\hline ceftriaxone ( $3^{\text {rd }}$ cephalosporins) & - & 1 & - & 1 \\
\hline ofloxacin (fluoroquinolones) & - & 1 & - & 1 \\
\hline norfloxacin (fluoroquinolones) & - & 1 & - & 1 \\
\hline sulfasalazine (sulfonamides) & 1 & - & - & 1 \\
\hline metronidazole (antiamoebic) & 1 & - & - & 1 \\
\hline clofazimine (antileprotic) & - & 1 & - & 1 \\
\hline isoniazid (antitubercular drug) & - & 1 & - & 1 \\
\hline artesunate (antimalarial) & 1 & - & - & 1 \\
\hline efavirenz (antiretroviral) & - & 1 & - & 1 \\
\hline Anticonvulsants & [6] & [7] & - & $13(28.89 \%)$ \\
\hline phenytoin & 3 & 4 & - & 7 \\
\hline sodium valproate & 2 & 1 & - & 3 \\
\hline carbamazepine & 1 & 2 & - & 3 \\
\hline NSAIDs & {$[1]$} & {$[1]$} & {$[1]$} & $3(6.67 \%)$ \\
\hline etoricoxid & 1 & - & - & 1 \\
\hline aspirin & - & - & 1 & 1 \\
\hline ibuprofen & - & 1 & - & 1 \\
\hline Antipyretics & {$[4]$} & [4] & - & $8(17.78 \%)$ \\
\hline paracetamol & 4 & 4 & - & 8 \\
\hline Antihistaminics & [1] & - & - & $1(2.2 \%)$ \\
\hline $\begin{array}{l}\text { Sinarest (chlorpheniramine maleate } 2 \\
\text { mg, phenylephrine hydrochloride } 10 \mathrm{mg} \text {, } \\
\text { paracetamol } 500 \mathrm{mg} \text {, anhydrous caffeine } 30 \mathrm{mg} \text { ) }\end{array}$ & 1 & - & - & 1 \\
\hline Antigout agent & -- & {$[1]$} & - & $1(2.2 \%)$ \\
\hline allopurinol & - & 1 & - & 1 \\
\hline Anticancer drugs & [2] & - & - & $2(4.4 \%)$ \\
\hline lenalidomide & 1 & - & - & 1 \\
\hline methotrexate & 1 & - & - & 1 \\
\hline Antidepressant & - & [1] & - & $1(2.2 \%)$ \\
\hline reboxetine & - & 1 & - & 1 \\
\hline Total & 19 & 25 & 1 & 45 \\
\hline
\end{tabular}

One case of SJS-TEN overlaps was reported due to aspirin. Sinarest tablet containing chlorpheniramine maleate $2 \mathrm{mg}$, phenylephrinehydrochloride $10 \mathrm{mg}$, paracetamol $500 \mathrm{mg}$, and anhydrous caffeine $30 \mathrm{mg}$ was taken over-the-counter by a patient and induced SJS. While considering individual drugs, paracetamol $(8 / 45,17.77 \%)$ \& phenytoin $(7 / 45,15.55 \%)$ were among the commonly reported offending drugs (Table 1). The conditions for which patients took these offending drugs were seizure, high-grade fever, arthralgia, cerebrovascular accident, multiple myeloma, chronic diarrhoea, ovarian tumour, HIV, pulmonary tuberculosis, hansen's disease, malaria, cystic kidney disease, cervical spondylosis, paranoid schizophrenia, gout and depression.
Various complications were observed in SJS, TEN and SJS-TEN overlap patients. Liver function test $(20 / 45,44.44 \%)$ followed by leukocytosis $(15 / 45$, $33.33 \%)$, neutrophilia $(10 / 45,22.22 \%)$, leukocytopenia $(5 / 45,11.11 \%)$, eosinophilia $(5 / 45,11.11 \%)$, neutropenia $(3 / 45,6.66 \%)$, thrombocytopenia $(3 / 45,6.66 \%)$, hypokalemia $(2 / 45,4.44 \%)$, and hyperglycemia $(1 / 45$, $2.22 \%$ ) were reported. Other complications were secondary infections, ocular, electrolytes imbalance, gastritis, oral candidiasis, pulmonary edema, respiratory distress, lung abscess, psychosis, septicaemia, and acute renal failure. In treatment, almost all patients have received systemic steroid comprised of dexamethasone $(29 / 45,64.44 \%)$, prednisolone $(14 / 45,31.11 \%)$, and prednisolone and dexamethasone pulse therapy $(2 / 45,8.88 \%)$. 


\section{DISCUSSION}

In our study, male preponderance (55.6\% male and $44.4 \%$ female) was observed, unlike female preponderance from previous studies. ${ }^{12-14}$ The mean age in previous studies were varied from 22 to 50 year ${ }^{5,15-18}$ whereas in our study, most of the patients (62.22\%) were beyond 21 year of age and mean age was 29.69 year. Duration between the drug intake and first onset of symptom in SJS/TEN ranges between few hours and 45 days, and the period of hospitalization ranges from 5 to 31 days. ${ }^{19}$ In our study, the median time duration between drug intake and onset of symptoms (25), and the median duration of hospital stay (13) were within these ranges.

In several studies, antimicrobials, anticonvulsants and NSAIDs were the most common group of drugs causing SJS, TEN and SJS-TEN overlap. ${ }^{15-19}$ Similarly in our study, antimicrobials were commonly reported group of drugs followed by anticonvulsants, antipyretics, and NSAIDs. Sixteen patients (35.5\%) had experienced SIS/TEN due to antimicrobials. The majority of TEN cases (44\%) were due to antimicrobials which was in concordance with the studies from (42\%) West Germany ${ }^{13}$ \& (44\%) South India. ${ }^{19}$ Penicillins and cephalosporins were the commonly reported causative drugs from Japan \& Singapore. ${ }^{17,18}$ In present study, among $\beta$-lactam antibiotics, cephalosporins were implicated in 4 cases. Fluoroquinolones were commonly reported from Indian state, Gujarat \& Karnataka. ${ }^{2,5}$ Antibacterial sulphonamides were the most common offending drugs reported from Lome, Togo. ${ }^{15}$ In our study, 3 cases were reported due to fluoroquinolones (ofloxacin, norfloxacin, and levofloxacin) and 2 cases were reported due to sulphonamides (sulfamethizole and sulfasalazine).

Anticonvulsants (28.89\%) were second most commonly reported offending drugs for SJS/TEN. Among them, phenytoin was the commonly reported offending drug followed by carbamazepine and sodium valproate. Phenytoin and carbamazepine are most common antiepileptic drugs reported for SJS/TEN in Asian population. ${ }^{20}$ As reported, the risk of developing SJS/TEN in patients who carries HLA-B ${ }^{\star} 1502$ allele is very high. ${ }^{21}$ Therefore, drug controller authority of India has instructed manufacturers to include SJS in package inserts of the product containing carbamazepine and advised to screen the patients for HLA-B ${ }^{\star} 1502$ allele before initiating treatment with carbamazepine. ${ }^{9}$ Sodium valproate is rarely reported as an offending drug in literature ${ }^{2}$ whereas in present study, 3 cases were reported due to sodium valproate. Eight cases of SJS/TEN were reported due to paracetamol which was in concordance with the study from Gujarat, India. ${ }^{2}$ NSAIDs were commonly reported offending drugs from France. ${ }^{22}$ In present study, 3 cases of SJS/TEN were reported due to NSAIDs (etoricoxib, aspirin, and ibuprofen). It was observed that patients often took an analgesic or antipyretic agent to treat unspecific symptoms such as fever or pain, which might be early sign of SJS/TEN or indication for an infection. Therefore, there was always doubt for any causal relation for analgesics or antipyretics. HIV is a most common co-morbid condition in the patients of
SJS/TEN ${ }^{2}$ and mortality is also very high in these patients. ${ }^{15}$ Among antiretrovirals, nevirapine is commonly reported causative drug ${ }^{15,19}$ whereas in this study 1 case of efavirenz-induced TEN was reported. HLA- ${ }^{\star} 5801$ allele is another genetic marker reported to have strong association with allopurinol-induced SJS/TEN..$^{21}$ In Europe \& Asia, allopurinol is commonly reported offending drug for SJS \& $\mathrm{TEN}^{18}$ whereas in present study, only 1 case of allopurinol-induced TEN was reported. Few cases of SJS/TEN due to antituberculars (isoniazid), cytotoxic drugs (lenalidomide, methotrexate), antimalarials (artesunate), antileprotics (clofazimine), antiamoebic (metronidazole) and antidepressant (reboxetine) were also reported in our study. Considering their widespread use across India, risk seems minimal.

The most common complications in SJS/TEN patients are septicemia, secondary infections, pneumonitis and acute renal failure. ${ }^{2}$ In our study, secondary infection was the most common complication in SJS/TEN patients. Beside this, altered liver functions, hematological, electrolytes, hyperglycemia and renal functions were also the conditions reported in this study.

It was observed that the use of ALDEN requires good data. This algorithm is specific to SJS and TEN because it includes some characteristic that are specific to these disease. A precise clinical history of the patient is needed to determine the index day and to document alteration in liver and kidney function because these are taken into account in determining the presence of the drug in the body at the time of onset of the adverse reaction. ${ }^{10}$ In our study, as per ALDEN, drug causality was very probable in 16 cases and was probable in 14 cases. However, only 1 case was certain and 29 cases were probable by WHO-UMC system.

\section{CONCLUSION}

Antimicrobials, anticonvulsants and antipyretics were commonly reported offending group of drugs for SJS/TEN in patients. Paracetamol $(8 / 45)$ \& phenytoin (7/45) were commonly reported offending drugs in individual drug category. Most of the cases were categorized very probable as per ALDEN whereas by WHO-UMC causality assessment system most of the cases were probable. Since the number of cases studied is less, it warrants further research.

\section{ACKNOWLEDGEMENTS}

Authors would like to thank post graduate trainees of Department of Dermatology \& STD, Gauhati Medical College \& Hospital, Guwahati-32, Northeast India for reporting adverse drug reactions. Authors also like to acknowledge National Coordination Centre-Pharmacovigilance programme of India, Indian Pharmacopoeia Commission, Ministry of Health \& Family Welfare, Govt. of India for permitting us to conduct this study.

\section{CONFLICT OF INTEREST}

The author declare no conflict of interest.

\section{ABBREVIATIONS USED}

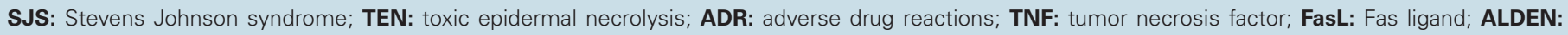

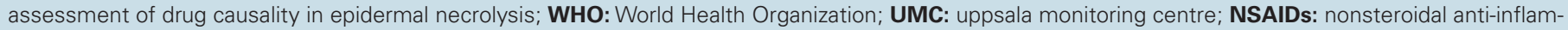
matory drugs.

\section{ABOUT AUTHOR}

Ratan J. Lihite: is a Technical Associate under Indian Pharmacopoeia Commission, Ministry of Health and Family Welfare, Govt. of India posted at ADR Monitoring Centre, Department of Pharmacology, Gauhati Medical College, Guwahati-32, Assam, Northeast India. He did his post graduation from Department of Pharmacy Practice, National Institute of Pharmaceutical Education and Research (NIPER)-Guwahati, Northeast India and has well experience in the area of pharmacovigilance and clinical pharmacology. Certificate of appreciation was bestowed by Drugs Controller General of India (DCGI), Hon'ble Dr. G.N. Singh for his valuable contribution in the Pharmacovigilance Programme of India (PvPI) at Indian Pharmacopoeia Commission in 2014. 


\section{REFERENCES}

1. Svensson CK, Cowen EW, Gaspari AA. Cutaneous Drug Reactions. Pharmacological Reviews. 2001;53(3):357-79.

2. Patel TK, Barvaliya MJ, Sharma D, Tripathi C. A systematic review of the druginduced Stevens-Johnson syndrome and toxic epidermal necrolysis in Indian population. Indian J Dermatol Venereol Leprol. 2013;79(3):389-98.

3. Harr T, French LE. Toxic epidermal necrolysis and Stevens-Johnson syndrome. Orphanet J Rare Dis. 2010;5(1):39.

4. Saha K. Toxic epidermal necrolysis: Current concepts in pathogenesis and treatment. Indian Dermatol Venereol Leprol. 2000;66(1):10-7.

5. Naveen KN, Pai W, Rai V, Athanikar SB. Retrospective analysis of Steven Johnson syndrome and toxic epidermal necrolysis over a period of 5 years from northern Karnataka, India. Indian J Pharmacol. 2013;45(1):80-2.

6. Wolkenstein $\mathrm{P}$, Revuz J. Drug-induced severe skin reactions. Incidence, management and prevention. Drug Saf. 1995;13(1):56-68.

7. Mokenhaupt M, Schopf E. Epidemiology of drug-induced severe skin reactions. Semin Cutan Med Surg. 1996;15(4):236-243

8. Bastuji-Garin S, Rzany B, Stern RS, Shear NH, Naldi L, Raujeau JC. Clinical classification of cases of toxic epidermal necrolysis, Stevens Johnson Syndrome and erythema multiforme. Arch Dermatol. 1993;129(1):92-6.

9. Lihite RJ, Lahkar M. An update on Pharmacovigilance Programme of India. Front Pharmacol. 2015;6:194.

10. Ameen KH, Pinninti R, Jami S. Aceclofenac induced Stevens-Johnson/toxic epidermal necrolysis overlap syndrome. J Pharmacol Pharmacother. 2013;4(1): 69-71.

11. Mockenhaupt M, Viboud C, Dunant A, Naldi L, Halevy S, Bouwes BJN, et al. Steven-Johnson syndrome and toxic epidermal necrolysis: assessment of medication risks with emphasis on recently marketed drugs. The Euro SCARstudy. J Invest Dermatol. 2008;128(1);35-44.

12. Devi K, George S, Criton S, Suja V, Sridevi PK. Carbamazepine-The commonest cause of toxic epidermal necrolysis and Stevens-Johnson syndrome: A study of 7 years. Indian J Dermatol Venereol Leprol. 2005;71(5):325-8.
13. Schopf E, Stuhmer A, Rzany B, Victor N, Zentgraf R, Kapp JF. Toxic epidermal necrolysis and Stevens-Johnson syndrome: An epidemiologic study from West Germany. Arch Dermatol.1991;127(6):839-42.

14. Lihite RJ, Lahkar M. A Study on Cutaneous Adverse Drug Reactions in ADR Monitoring Centre of Tertiary Care Hospital, Guwahati. J App Pharm Sci. 2013;3(3):79-81

15. Saka B, Kombate K, Mouhari-Toure A, Akakpo S, Tchangai-Walla K, Pitche P. Stevens-Johnson syndrome and toxic epidermal necrolysis in a teaching hospital in Lome, Togo: retrospective study of 89 cases. Med Trop (Mars). 2010;70(3):255-8

16. Chen J, Wang $B$, Zeng $Y, X u H$. High-dose intravenous immunoglobulins in the treatment of Stevens-Johnson syndrome and toxic epidermal necrolysis in Chinese patients: a retrospective study of 82 cases. Eur J Dermatol. 2010;20(6):743-7.

17. Yamane $Y$, Aihara M, Ikezawa Z. Analysis of Stevens-Johnson syndrome and toxic epidermal necrolysis in Japan from 2000 to 2006. Allergol Int. 2007;56(4): 419-25.

18. Tan SK, Tay YK. Profile and pattern of Stevens-Johnson syndrome and toxic epidermal necrolysis in a general hospital in Singapore: treatment outcomes. Acta Derm Venereol. 2012;92(1):62-6.

19. Sanmarkan AD, Sori T, Thappa DM, Jaisankar TJ. Retrospective Analysis of Stevens-Johnson syndrome and Toxic Epidermal Necrolysis over a Period of 10 Years. Indian J Dermatol. 201:56(1):25-9.

20. Yang $\mathrm{CY}$, Dao RL, Lee TJ, Lu CW, Yang $\mathrm{CH}$, Hung $\mathrm{SI}$, et al. Severe cutaneous adverse reactions to antiepileptic drugs in Asians. Neurology. 2011;77 (23):2025-33.

21. Chung WH, Hung SI. Genetic Markers and Danger Signals in Stevens-Johnson syndrome and Toxic Epidermal Necrolysis. Allergology International. 2010 59(4):325-32.

22. Roujeau JC, Guillaume JC, Fabre JP, Penso D, Fléchet ML, Girre JP. Toxic epiderma necrolysis (Lyell Syndrome): Incidence and drug etiology in France, 1981-1985. Arch Dermatol. 1990;126(1):37-42. 